\title{
Event-based speed control on a sensor-less miniature thruster
}

\author{
Thibaut Raharijaona $^{1}$, Lorris Dola ${ }^{1}$, Bruno Boisseau ${ }^{2}$, John-Jairo Martinez-Molina ${ }^{2}$, Nicolas Marchand ${ }^{2}$ \\ and Franck Ruffier ${ }^{1}$
}

\begin{abstract}
The main contribution of this paper is the experimental evaluation of the performance of different event-based control strategies in the case of a sensor-less miniature thruster.

While most control engineering applications considers periodic control with equidistant sample intervals, the event-based approach updates the control signal only when required. The performances in terms of thruster power consumption and control update number are evaluated by comparing the eventbased control system with respect to the time-based loop for the angular speed regulation of a sensor-less miniature thruster. The experimental results clearly show an important reduction of the control updates while the power consumptions are similar.
\end{abstract}

\section{INTRODUCTION}

Due to the necessity of saving the energy and increasing the autonomy of embedded systems, event-based control becomes an interesting research topic. While the classical socalled time-based framework of controlled systems consists in sampling the system uniformly in time with a constant sampling period and in computing and updating the control law every time instants, the event-based approach aims at updating asynchronously the control signal. The control signal is updated only when required. Some works addressed more recently event-based sampling where the control law is event-driven [1], [2], [3], [4].

For an application point of view, there are several possible ways to save the energy and improve the autonomy. The first way aims at reducing the computation load and the second way tends to reduce the actuation load. Low-power electronic components in all embedded and miniaturized mechatronic applications encourages companies to develop asynchronous versions of the existing time-triggered components. A significant power consumption reduction can be achieved by decreasing the samplings and consequently the CPU utilization. In [5], an event-based proportional integral derivative (PID) controller is presented with the simulation results applied to a double tank process. For stability reason, a maximal sampling period is introduced in the initial approach. In [6], this maximal period is removed and different event-based PID algorithms without safety limit condition were also developed. In [7], the efficiency of an approach without safety limit condition is highlighted by implementing such a controller in a real-time testbed. A radio-controlled vehicle embeds an event-based cruise control mechanism. In

\footnotetext{
${ }^{1}$ Thibaut Raharijaona, Lorris Dola and Franck Ruffier are with AixMarseille Université, CNRS, ISM UMR 7287, 13288, Marseille cedex 09, France thibaut.raharijaona@univ-amu.fr

${ }^{2}$ Bruno Boisseau, John-Jairo Martinez-Molina and Nicolas Marchand are with GIPSA-lab, Control Systems Department, UMR CNRS 5216-INPGUJF, ENSIEG BP 46, 38402 Saint Martin d'Heres Cedex, France
}

addition, the reduction of computational load is investigated in [8] and an event-driven system theory is drawn for disturbed linear systems.

In this paper, using miniature actuators which equipped the bio-inspired robot LORA III [9], an experimental setup was developed to test some event-based techniques for speed tracking and disturbance rejection. This work aims at comparing the performances of time-based and event-based control laws in terms of power consumption and control updates in particular an event-based state feedback approach that uses the instantaneous current information to limit the starting peak current and the power consumption. The paper is structured as follows. Firstly, the experimental platform is presented in section II. The event-based angular speed control laws of the miniature thruster are designed in section III. Experimental results are provided in section IV.

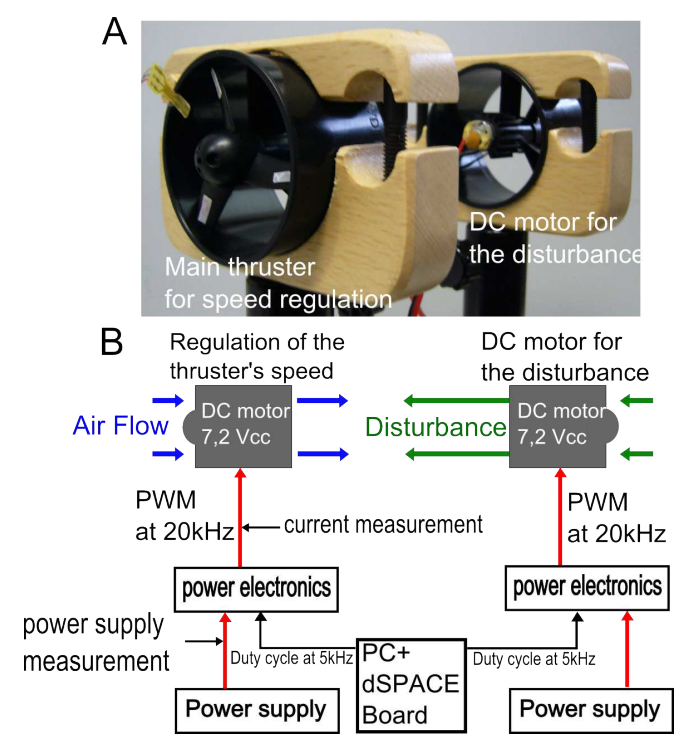

Fig. 1. A: Picture of the experimental platform which is equipped with a miniature thruster used to compare the event-based algorithms and a additional thruster which generates wind disturbance B: Sketch of the sensor-less miniature thruster testbed using a dSPACE electronic board

\section{THE MINIATURE SENSOR-LESS THRUSTER TESTBED}

The experimental platform is depicted in Fig. 1A. It is based on a miniature direct current thruster GWS EDF50 (see Fig. 1A, power: $24.41 \mathrm{~W}$, mass: $29 \mathrm{~g}$ ) which was previously embedded on the bio-inspired robot LORA III [9]. The maximum allowable current drawn is 3.4A under 7.2V. Some extra components were added to make possible the sensor-less thruster speed regulation. 


\section{A. The testbed}

The aim of the experimental set-up is to implement different kinds of time-based and event-based control laws from the literature for thruster's speed regulation and disturbance rejection without any angular speed measurement. The performances in term of power consumption and control updates are considered. As presented in the right side of Fig. $1 \mathrm{~B}$, a second thruster generates wind disturbances.

\section{B. The data acquisition and control}

The data acquisition and control are performed in a realtime framework using a dSPACE electronic board which is connected to the experimental platform. The control laws are implemented using Matlab/Simulink. The measurements and performances are monitored online and one can analyze them off line.

\section{Estimation of the rotor speed}

In this section, we propose to estimate the rotor speed. It simplifies mechanical assemblies and reduces the size of electronic circuits. Moreover, one can significantly reduce the sampling frequency $F_{s}=20 \mathrm{kHz}$ required if a reflective sensor would measure the thruster's angular speed. Therefore estimating the thruster's angular speed allows to save energy and computational resources. The speed estimation simply requires that the armature voltage and the motor current be measured. The estimation principle is presented in [10]. One can recall the main steps.

The dynamics of a DC motor is described by the following equations:

$$
\begin{gathered}
\quad \frac{d i(t)}{d t}=-\frac{R}{L} \cdot i(t)-\frac{K_{e} \cdot M}{L} \cdot \omega_{h}+\frac{1}{L} \cdot u(t) \\
\frac{d \omega_{h}(t)}{d t}=\frac{K_{t}}{J \cdot M} \cdot i(t)-\frac{D}{J \cdot M^{2}} \cdot \omega_{h}(t)-\frac{1}{J \cdot M} \cdot c_{0}(t) \\
\text { - } \omega_{h}(t): \text { the thruster angular speed }(\mathrm{rad} / \mathrm{s}) \\
\text { - } c_{0}(t): \text { an additive bounded disturbance } \\
\text { - } u(t): \text { the armature voltage }(\mathrm{V}) \\
\text { - } i(t): \text { the armature current }(\mathrm{A})
\end{gathered}
$$

The parameters are defined as:

- $D$ : the drag coefficient of the thruster (N.m.s/rad)

- $J$ : the inertia of the motor $\left(\mathrm{kg} \cdot \mathrm{m}^{2}\right)$

- $K_{t}$ : the torque constant (N.m/A)

- $K_{e}$ : the back-EMF voltage constant (V.s/rad)

- $L$ : the inductance $(\mathrm{H})$

- $M$ : the reduction ratio between the thruster and the rotor

- $R$ : the armature resistance $(\Omega)$

In the following, we consider that the mechanical time constant is greater than the electrical time constant. Therefore, we assume that the armature current $i(t)$ is constant during the thruster's speed $\omega_{h}(t)$ transient phase. As shown in Fig. 2A, the measurements of the armature voltage $u(t)=$ $v_{b a t}-v_{1}$ and the armature current $i(t)=\frac{v_{1}-v_{2}}{R_{s}}$ measured thanks to a current shunt resistor $R_{s}$, gives an estimated thruster's speed $\omega_{\text {est }}(t)=\hat{\omega}_{h}(t)$ in Volt such that : $\omega_{\text {est }}(t)=$
$u(t)-R_{s} i(t)$. In Fig. 2B, the measured current $i(t)$ is noisy and it is not possible to use it for estimation. The current measurement is filtered using a Kalman filter whose the process noise covariance is set 0.0034 and the measurement noise covariance is set to 20 after experiments. The measured current and the estimated current using the Kalman filter are shown in Fig. 2B.

In the Laplace domain, one writes: $\Omega_{e s t}(s)=U(s)-$ $R_{s} I(s)$ and from (1) in steady state, $U(s)=K_{e} M \Omega_{h}(s)+$ $R I(s)$. From (2), assuming $D$ is small and $c_{0}(t)=0$, in the Laplace domain $I(s)=\frac{s J M}{K_{t}} \Omega_{h}(s)$. Finally, the estimated velocity is written such as:

$$
\begin{gathered}
\Omega_{e s t}(s)=K_{e} M \Omega_{h}(s)+R I(s)-R_{s} I(s) \\
\Omega_{e s t}(s)=K_{e} M\left(1+\frac{s J\left(R-R_{s}\right)}{K_{e} K_{t}}\right) \Omega_{h}(s)
\end{gathered}
$$

We define $\tau=\frac{J\left(R-R_{s}\right)}{K_{e} K_{t}}$. In practice, one can remark that $\tau \ll 1$, therefore $\Omega_{e s t}(s) \simeq K_{e} M \Omega_{h}(s)$.

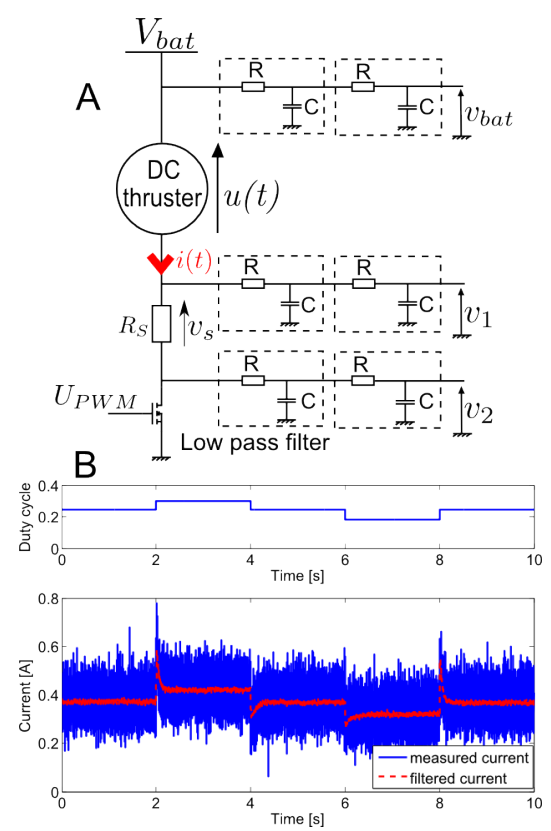

Fig. 2. A: Electric diagram of the DC thruster. The current $i(t)$ is obtained with the measured voltages $v_{1}$ and $v_{2}$. The voltages are filtered with a lowpass filter. The voltage $U_{P W M}$ controls the thruster speed through the transistor B: The current $i(t)$ is filtered with a Kalman filter. The top plot is the duty-cycle applied in open-loop and the bottom plot shows the measured current in blue line and the filtered current in red dashed line.

In Fig. 3, the estimated speed in dashed red line is compared to the measured speed in solid blue line using a reflective sensor at the sampling frequency $F_{s}=20 \mathrm{kHz}$. One can remark that the speed estimation is fair. The gain $K_{e} M$ from (3) is experimentally tuned and fixed for the following work. Finally, we estimate the thruster's speed without any velocity sensor using only the following measurements $u(t)$, $v_{1}(t)$ and $v_{2}(t)$. The estimated speed will feed the controllers for regulation and disturbance rejection at a lower sampling frequency $F_{s}=5 k H z$. 

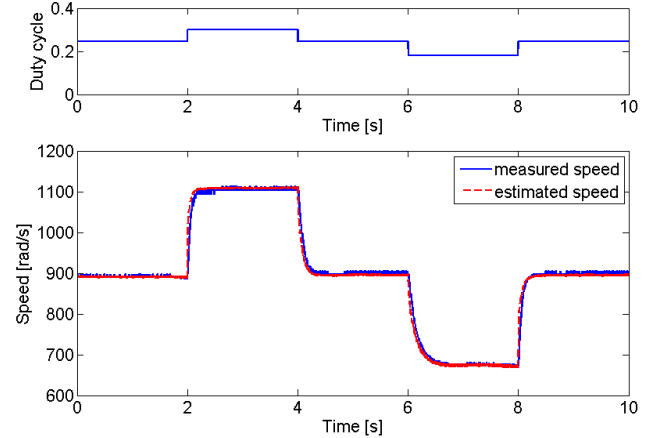

Fig. 3. Comparison between the measured speed and the estimated speed the top plot is the duty-cycle applied in open-loop and the bottom plot shows the measured speed in blue line and the estimated speed in red dashed line.

\section{Discrete-time model identification}

Under the assumption of homogenous magnetic field, the miniature direct current thruster is supposed to be a linear plant. Equations (1) and (2) are considered. The variable to be controlled is the angular speed $\omega_{h}(t)$ which is estimated as detailed in sec. II-C. In addition, state-space oriented control is investigated in order to limit the starting peak current. Therefore, we define as state variables the armature current $i(t)$ and the thruster speed $\omega_{h}(t)$ such as:

$$
\left\{\begin{array}{l}
x(k+1)=A \cdot x(k)+B \cdot u(k) \\
y(k)=C \cdot x(k)
\end{array}\right.
$$

where $x(k)=\left[i(k), \omega_{h}(k)\right]^{T}, u(k)$ is the control input signal, $y(k)$ is the measurement, $\mathrm{A}$ is a $2 \times 2$ matrix, $\mathrm{B}$ is a $2 \times 1$ matrix and $C=\left[\begin{array}{ll}1 & 0 \\ 0 & 1\end{array}\right]$.

For the discrete time state-space model identification, data are recorded at the sampling frequency $F_{s}=5 \mathrm{kHz}$. The measured current varies from $0.2 \mathrm{~A}$ to $0.5 \mathrm{~A}$ and the estimated thruster's speed varies from approximately less than 900 $\mathrm{rad} / \mathrm{s}$ to $1100 \mathrm{rad} / \mathrm{s}$. The identified state-space model for the miniature thruster is given by the representation:

$$
\left\{\begin{array}{rr}
x(k+1)=\left[\begin{array}{cc}
0.9873 & 0 \\
-4.262 \times 10^{-5} & 0.9541
\end{array}\right] . x(k) \\
+\left[\begin{array}{c}
49.18 \\
0.2022
\end{array}\right] . u(k) \\
y(k)= \\
{\left[\begin{array}{cc}
1 & 0 \\
0 & 1
\end{array}\right] . x(k)}
\end{array}\right.
$$

For the state space model identification, the best fits for the speed and the current are respectively equal to $87.7 \%$ and $62.2 \%$.

The model validation is performed using a new set of data. Figures $4 \mathrm{~A}$ and $4 \mathrm{~B}$ exhibit the results. The fits for the speed and the current are respectively equal to $88.7 \%$ and $61.6 \%$.

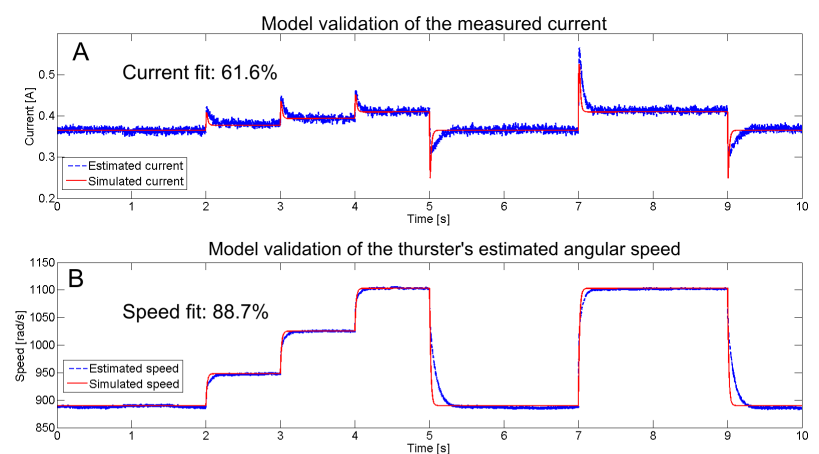

Fig. 4. Comparison between the measured outputs and the simulated model outputs with the same input. A: Comparison between the estimated current in dashed blue line and the simulated current in red solid line. B: Comparison between the estimated speed in dashed blue line and the simulated speed in red solid line.

\section{EVENT-BASED SPEED CONTROL}

The thruster's speed is usually controlled with a timebased proportional-integral (PI) strategy or time-based state feedback. In this section, we propose to design some eventbased approaches introduced in [6] and [8] for both regulation and disturbance rejection and to compare the results to the ones obtained with the time-based controllers.

\section{A. Time-based PI control}

The proportional-integral controller in frequency domain is:

$$
U(s)=K_{p}\left[E(s)+\frac{1}{T_{i} s} E(s)\right]
$$

where $U($.$) is the control signal and E($.$) the error between$ the estimated output of the controller system and a given setpoint. $K_{p}$ and $T_{i}$ are some tunable parameters. A discrete time controller is finally obtained, that is:

$$
\left\{\begin{array}{l}
u_{P}=K_{p} \cdot\left(\omega_{r e f}(k)-\omega_{h}(k)\right) \\
u_{I}(k)=u_{I}(k-1)+\frac{K_{p}}{T_{i}} \cdot T_{s} \cdot\left(\omega_{r e f}(k)-\omega_{h}(k)\right) \\
u(k)=u_{p}(k)+u_{I}(k)
\end{array}\right.
$$

where $T_{s}=2 \cdot 10^{-4}$ s (ie: $F_{s}=5 k H z$ ).

In order to limit the amplitude of the control input and the peaks of current, we choose the same settling time at $95 \%$ for the closed-loop and the open-loop systems i.e. $\tau_{\text {settling }}=$ $0.173 \mathrm{~s}$. Then $T_{i}=0.0442 \mathrm{~s}$ and $K_{p}=2.9377 .10^{-4}$ are the parameters of the PI controller which gives a gain margin of $64.9 \mathrm{~dB}$ and a phase margin of $90^{\circ}$.

\section{B. Time-based state feedback control}

A discrete-time state feedback controller with integral action for trajectory tracking is also designed such that: $u(k)=-\left[\begin{array}{lll}K_{i} & K_{\omega_{h}} & K_{z}\end{array}\right] \cdot\left[\begin{array}{lll}i(k) & \omega_{h}(k) & z(k)\end{array}\right]^{T}$. Using a backward discretization, the discrete-time control signal is computed as follows:

$$
\left\{\begin{array}{l}
u_{P}(k)=-\left[K_{i} K_{\omega_{h}}\right] \cdot\left[i(k) \omega_{h}(k)\right]^{T} \\
u_{I}(k)=u_{I}(k-1)-K_{z} \cdot T_{s} \cdot\left(\omega_{r e f}(k)-\omega_{h}(k)\right) \\
u(k)=u_{P}(k)+u_{I}(k)
\end{array}\right.
$$


Where $K_{i}=-0.2270, K_{\omega_{h}}=-0.0001$ and $K_{z}=$ -0.0015 . The settling time at $95 \%$ is the same as for the PI controller.

\section{Event-based state feedback control}

The event-based state feedback controller is designed using [8]. A geometric interpretation is proposed in Fig. 5. The control update $u(k)$ is computed at a constant sampling rate $T_{s}$ when the state of the system lies outside a pre-defined set $\beta$. When the state is inside the set $\beta$, the control keeps its previous value. If we consider the system $x(k+1)=$ $A . x(k)+B \cdot u(k)$, we design the set $\beta$ where the control is held by $\beta:=\left\{x \in \mathbb{R}^{n}|| x \mid<e_{T}\right\}$. The threshold $e_{T}$ ensures a certain level of performance. The discrete-time state feedback control signal is computed as follows:

$$
u(k)=\left\{\begin{array}{l}
-K x(k) \text { if }|x(k)| \geqslant e_{T} \\
u(k-1) \text { if }|x(k)|<e_{T}
\end{array}\right.
$$

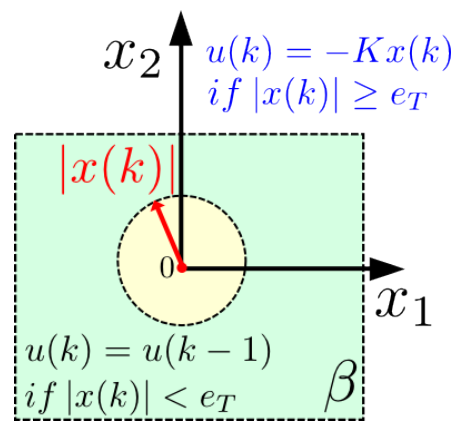

Fig. 5. Geometric interpretatrion of the event-based state feedback control proposed in [8]. As long as the state $x(k)$ is inside the set $\beta$, the control input signal is $u(k)=u(k-1)$.

Let introduce the instant $\tau_{k} . \tau_{k}$ denotes the event times by using a zero-order hold such that: $u(t)=u(k)$ for all $t \in\left[\tau_{k}, \tau_{k+1}\right)$. Therefore, $x(k)=x\left(\tau_{k}\right)$ and $u(k)=u\left(\tau_{k}\right)$. In this paper, the controller checks whether the state is inside or outside the set $\beta$ at a constant rate. This rate is chosen to be equal to the sampling rate of the controller $T_{s}$ such as $\tau_{k+1}=\tau_{k}+T_{s}$. Then as long as the state is outside the set $\beta$, the control is updated at a constant sampling time. When the state reaches the set $\beta$ the control is held to the value just before entering the set $\beta$. The gains $K_{i}, K_{\omega_{h}}$ and $K_{z}$ are the same as for the time-based state feedback controller.

\section{Event-based PI control without safety limit condition}

The event-based control described in [6] is designed. The event occurs when $|e(k)-e(k-1)|>e_{\text {lim }}$ where $e$ is the error $e(k)=\omega_{\text {ref }}(k)-\omega_{h}(k)$ and $e_{\text {lim }}$ is a pre-defined threshold. As proposed in [6], we implement an algorithm without the safety limit condition firstly introduced in [5] in order to prevent the output from high overshoot. The hybrid algorithm is a mix between the approach with an exponential forgetting factor and the approach with a saturation of the product $h_{a c t} . e$. The resulting algorithm is:

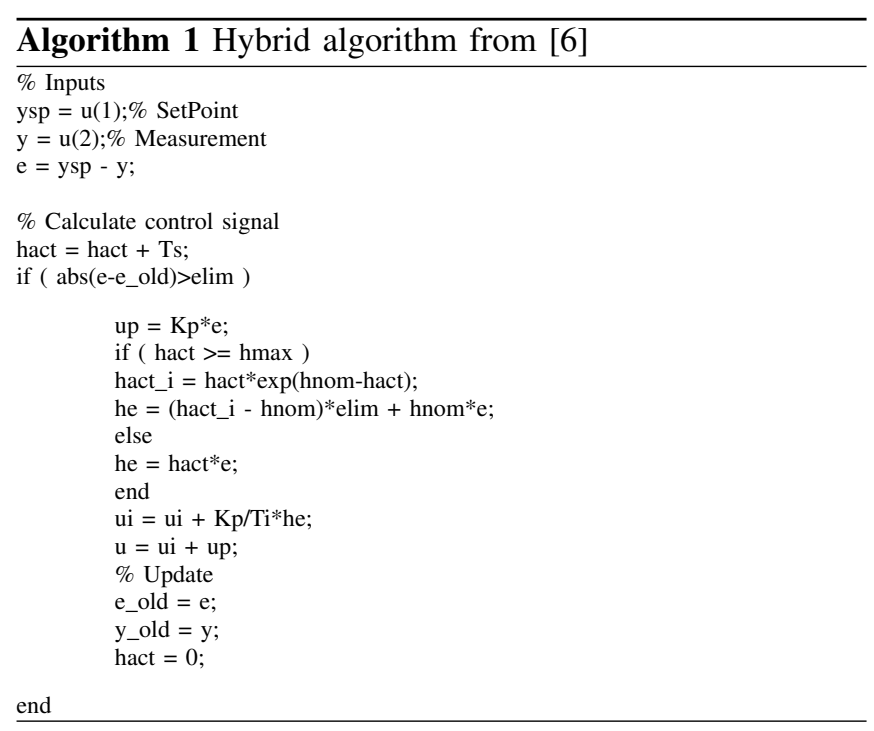

The parameters $h_{\max }$ and $e_{\text {lim }}$ are chosen such as the control update are significantly reduced while the control allows a reference tracking. Therefore, $h_{\max }=4$ and $e_{\text {lim }}=$ 0.1 . Moreover, the gain $K_{p}$ and $T_{i}$ are the same as the ones used for the time-based PI controller.

\section{EXPERIMENTAL RESULTS}

In this section, we propose to test the different eventbased control strategies and to compare their performances to the time-based approaches on the miniature thruster for trajectory tracking and disturbance rejection.

\section{A. Trajectory tracking}

In Fig. 6A, the thruster's speed with the event-based PI controller from Sec. III-D is plotted and is compared to the time-based PI controller. The event-based approach performs a trajectory tracking without static error. One can remark increases of the overshoots during the changes of reference with the event-based approach. In Fig. 6A, the trajectory tracking is performed with only 1778 updates which is a reduction of $96.5 \%$ of the control updates (see Fig. 6B). The power consumption is smaller with the event-based controller $(0.52 \mathrm{~W})$ than with the time-based one $(0.54 \mathrm{~W})$. Figures $6 \mathrm{C}$ and $6 \mathrm{D}$ show the comparison between the duty cycles of the PWM in $\%$ and the currents.

In Fig. 7A, the thruster's speed with the event-based state feedback controller from Sec. III-C is plotted and is compared to the time-based state feedback controller.

We define the performance set $\beta$ such that:

$$
\beta:=\left\{\begin{array}{l}
\omega_{\text {ref }}-\omega_{\text {tolerance }}<\omega_{h}<\omega_{\text {ref }}+\omega_{\text {tolerance }} \\
i_{\text {set }}-i_{\text {tolerance }}<i<i_{\text {set }}+i_{\text {tolerance }}
\end{array}\right.
$$

with $\omega_{\text {tolerance }}=40 \mathrm{rad} / \mathrm{s}, i_{\text {set }}=0.3 \mathrm{~A}$ and $i_{\text {tolerance }}=$ $0.1 A$. Figure $7 \mathrm{~A}$ shows that the event-based state feedback performs the reference tracking with a static error lower than $\omega_{\text {tolerance }}=40 \mathrm{rad} / \mathrm{s}$.

The reduction of the control updates is about $91 \%$ with the event-based state feedback (see Fig. 7B). 


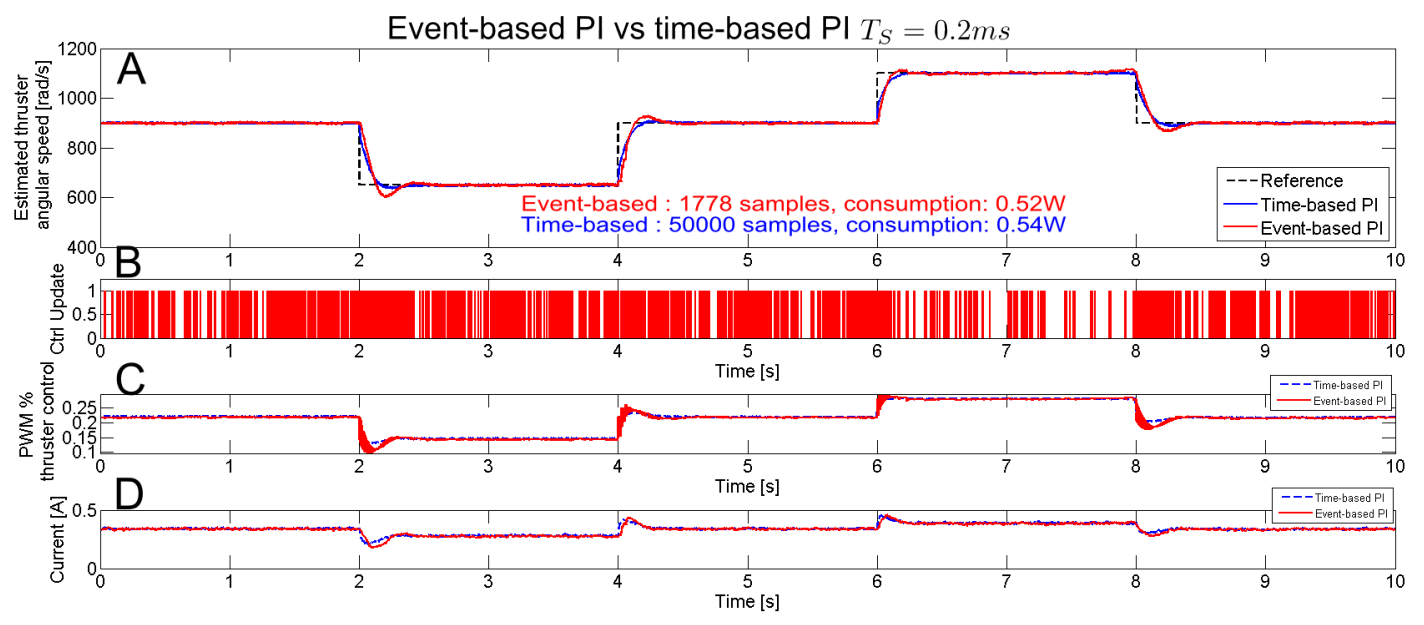

Fig. 6. A: Trajectory tracking: comparison between the time-based PI in dashed blue line and the event-based PI from [6] in red line. The figure shows the evolution of the thruster speed with both types of control. B: The event-based control update is also displayed. The update is performed when the indicator is at 1. C: The duty cycles of the PWM in \% with the time-based PI in dashed blue line and the event-based PI in red line. D: The current with the time-based PI in dashed blue line and the event-based PI in red line.

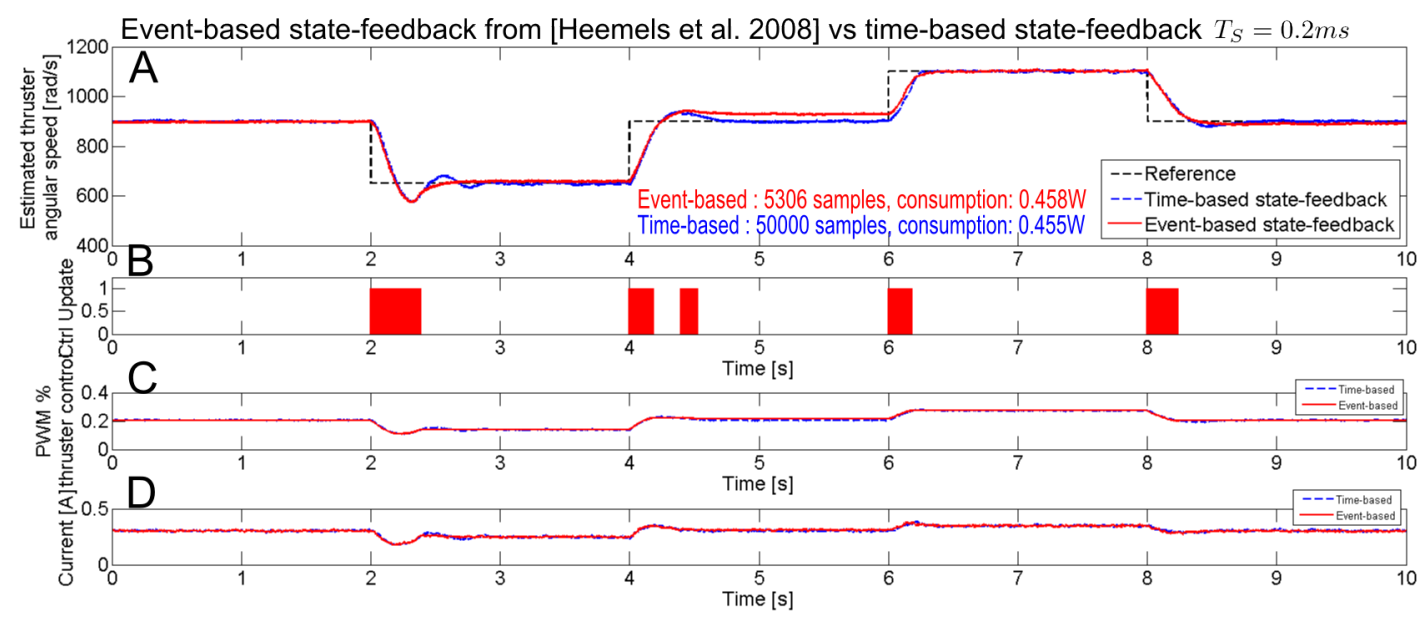

Fig. 7. A: Trajectory tracking: comparison between the time-based state feedback in dashed blue line and the event-based state feedback from [8] in red line. B: The event-based control update is also displayed. The update is performed when the indicator is at 1 . C: The duty cycles with the time-based state feedback in dashed blue line and the event-based state feedback in red solid line. D: The current with the time-based state feedback in dashed blue line and the event-based state feedback in red solid line.

The power consumptions are slightly identical $(0.45 \mathrm{~W})$. In Fig. $7 \mathrm{C}$, the duty cycle of the PWM is only updated during the changes of reference and keeps its previous value during the steady-state. Figure 7D displays the currents. The trajectories of the state $\omega_{h}$ versus $i$ with the time-based and event-based controllers are plotted in Fig. 8. One can see that the trajectory of the states converges to the different performance sets $\operatorname{Set}(A), \operatorname{Set}(B)$ and $\operatorname{Set}(C)$.

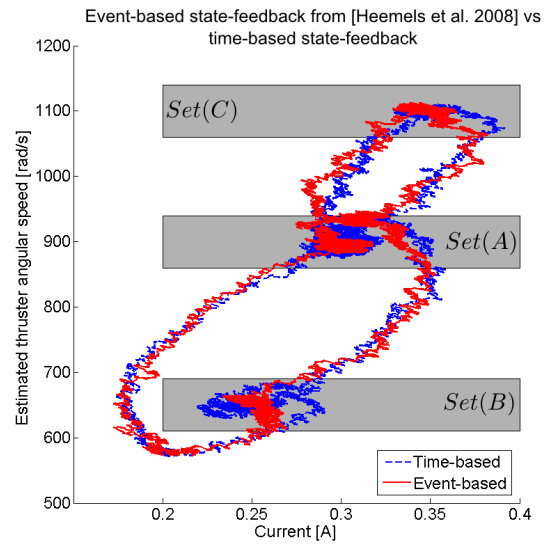

Fig. 8. The figure shows the phase portrait in closed loop: the current $i(t)$ and the thruster speed $\omega_{h}(t)$. The trajectories of the time-based controller and the event-based one are respectively plotted in dashed blue line and in red solid line. The performance sets are represented by the grey rectangles. 


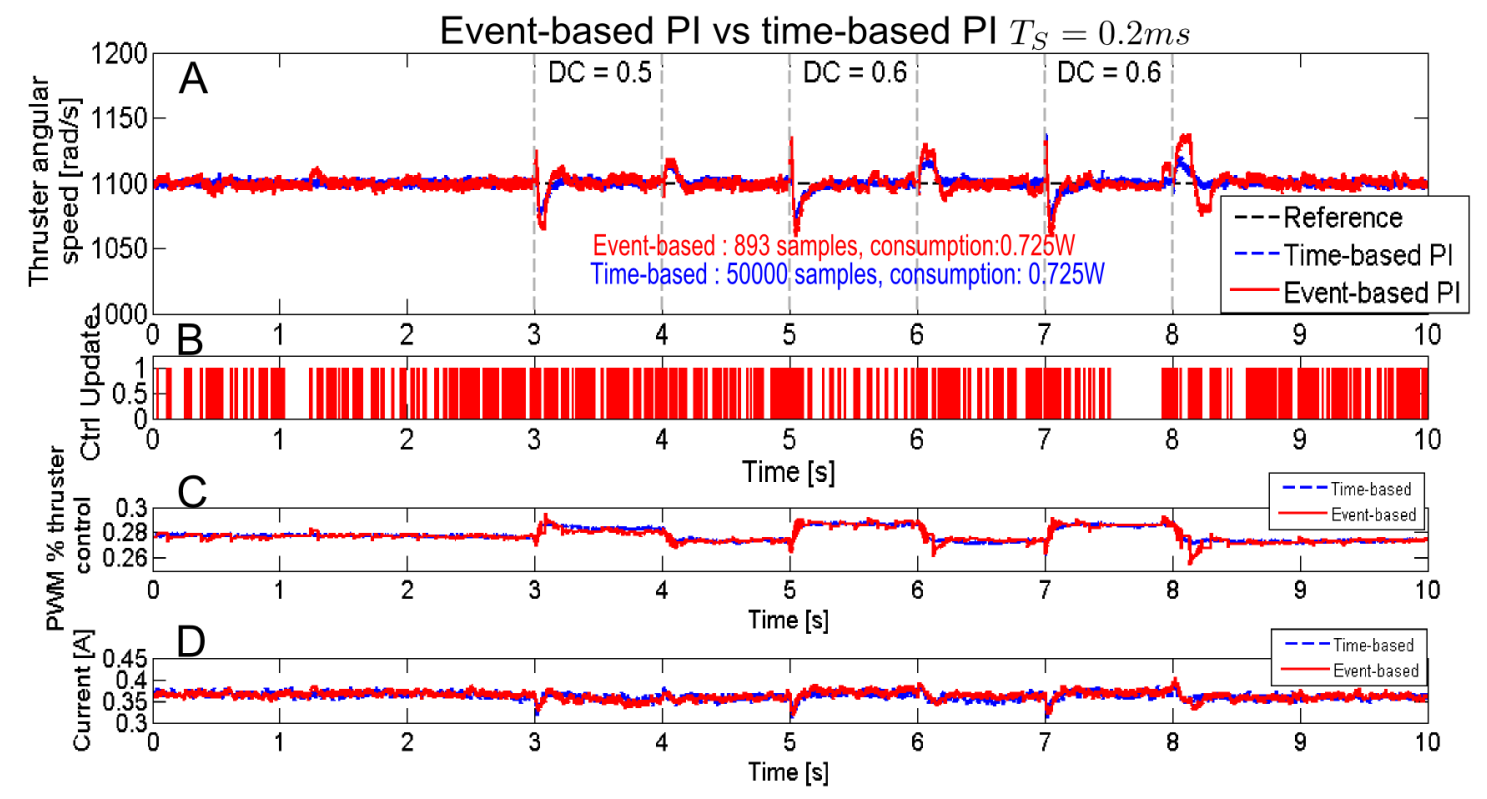

Fig. 9. A: Disturbance rejection: comparison between the time-based PI in dashed blue line and the event-based PI from [6] in red line. B: The event-based control update is also displayed. The update is performed when the indicator is at 1. C: The duty cycles of the PWM in \% with the time-based PI in dashed blue line and the event-based PI in red solid line. D: The current with the time-based PI in dashed blue line and the event-based PI in red solid line.

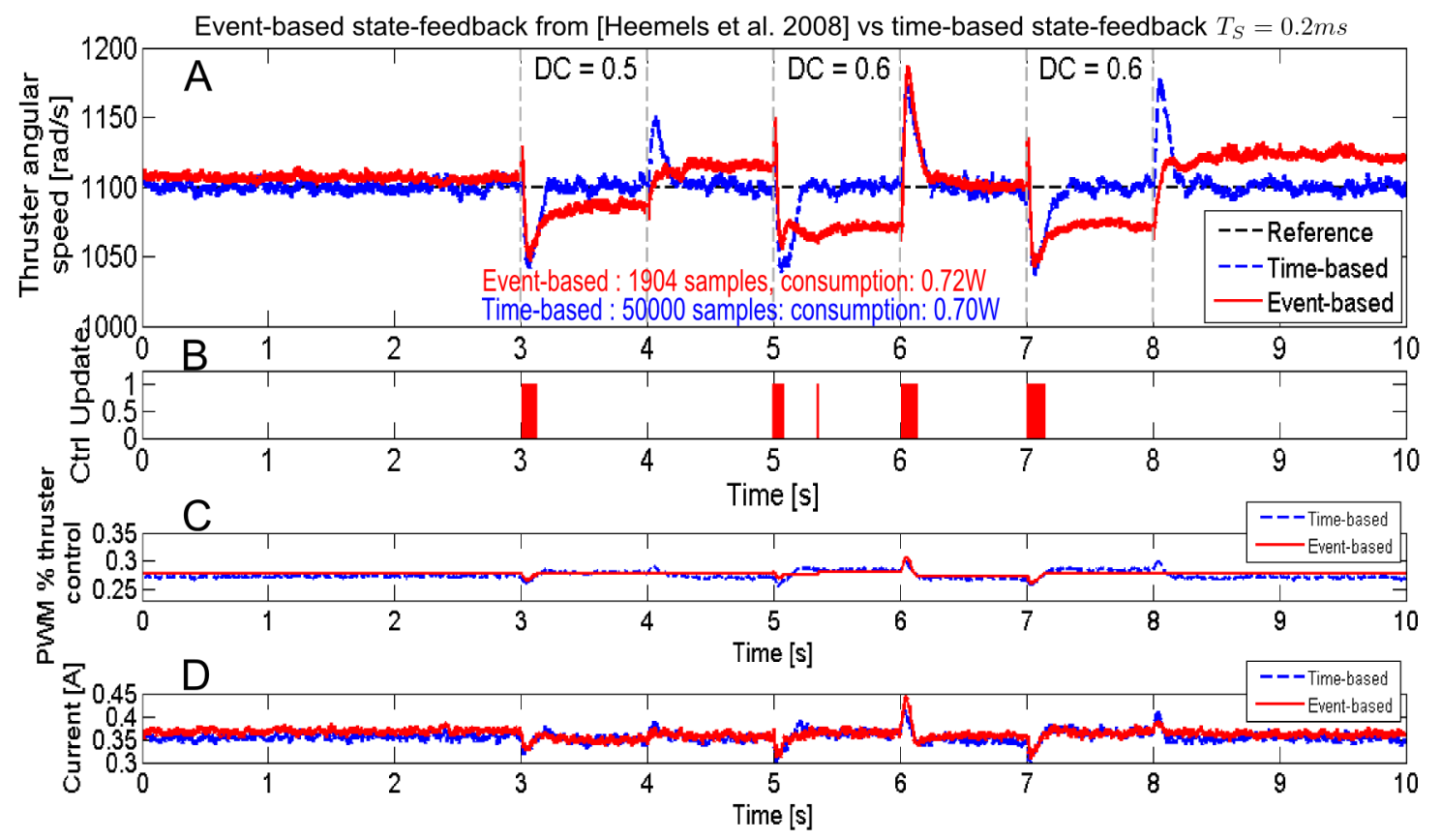

Fig. 10. A: Disturbance rejection: comparison between the time-based state feedback in dashed blue line and the event-based state-feedback from [8] in red line. B: The event-based control update is also displayed. The update is performed when the indicator is at 1 . C: The duty cycles with the time-based state feedback in dashed blue line and the event-based state feedback in red solid line. D: The current with the time-based state feedback in dashed blue line and the event-based state feedback in red solid line. 


\section{B. Disturbance rejection}

In this section the disturbances are gusts of wind generated by a second thruster. The latter is controlled in open-loop with different duty cycles and activated at different moments. The disturbances occurs at each interval $t \in[3 s ; 4 s], t \in$ $[5 s ; 6 s]$ and $t \in[7 s ; 8 s]$ with a duty cycle respectively equals to $0.5,0.6$ and 0.6 otherwise the motor is turned off with a duty cycle equal to 0 . The speed of the main thruster is regulated at $1100 \mathrm{rad} / \mathrm{s}$. Figure $9 \mathrm{~A}$ shows that the eventbased PI controller rejects the disturbances despite larger overshoots than the ones obtained with the time-based PI controller. The control updates are displayed in Fig. 9B, the reduction of the control updates is about $92 \%$. The power consumptions are the same with the time-based and the event-based controllers $(0.725 \mathrm{~W})$. The comparison of the control input signals is presented in Fig. 9C and Fig. 9D. From Fig. 10A, the event-based state feedback controller rejects the disturbances. The control updates are reduced up to $96 \%$ (see Fig. 10B). The duty cycle is only updated when the disturbances are added or removed (see Fig. 10C). Figure $10 \mathrm{D}$ plots the currents. The power consumptions are slightly the same with the time-based controller $(0.70 \mathrm{~W})$ and with the event-based controller $(0.72 \mathrm{~W})$.

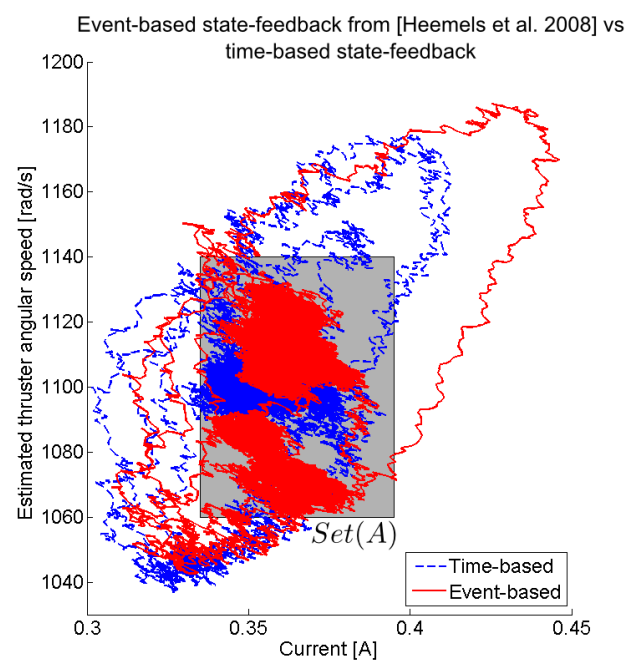

Fig. 11. The figure shows the phase portrait in closed loop: the current $i(t)$ and the thruster speed $\omega_{h}(t)$. The trajectories of the time-based controller and the event-based one are respectively plotted in dashed blue line and in red solid line. The performance set called $\operatorname{Set}(A)$ is represented by the grey rectangle.

Figure 11 shows the trajectories of the states under the disturbance rejection with the time-based and event-based state feedback controllers. As soon as the states are outside $\operatorname{Set}(A)$, the control is updated such that the states reach again the set. Once the control is held, the states converge inside the set. One can say that this strategy succeeds in limiting the power consumption and starting peak current by using the instantaneous current information.

\section{CONCLUSION}

An experimental platform was developed and aimed at testing some event-based control schemes using a miniature thruster. The event-based control laws performed a reference tracking and a disturbance rejection. The thruster's speed estimation was implemented thanks to the measurements of only three voltages. Therefore, the speed estimation was fed into the designed controllers. The event-based approach was performed with a PI controller and a state feedback controller. The event-based state feedback approach was based on a set defined by the controlled output and a inner state, the current, which is somehow an image of the instantaneous energy consumption. The event-based approaches allowed to significantly reduce the number of control updates while having a similar energy consumption compared to a timebased approaches while applying output disturbances.

\section{ACKNOWLEDGMENTS}

We thank M. Boyron for his assistance with the electrical design and J. Diperi for his involvement in the mechanical design of the testbed. This work is part of the project PEPS SpikeRob, supported by The French National Center for Scientific Research (CNRS).

\section{REFERENCES}

[1] K. Astrom and B. Bernhardsson, "Comparison of riemann and lebesgue sampling for first order stochastic systems," in Proceedings of the 41st IEEE Conference on Decision and Control, vol. 2, Dec 2002, pp. 2011-2016.

[2] J. H. Sandee, W. P. M. H. Heemels, and P. P. J. Van den Bosch, "Eventdriven control as an opportunity in the multidisciplinary development of embedded controllers," in Proceedings of the American Control Conference, vol. 3, June 2005, pp. 1776-1781.

[3] J. Lunze and D. Lehmann, "A state-feedback approach to event-based control," Automatica, vol. 46, no. 1, pp. $211-215,2010$ [Online]. Available: http://www.sciencedirect.com/science/article/pii/S0005109809004968

[4] A. Anta and P. Tabuada, "To sample or not to sample: Self-triggered control for nonlinear systems," IEEE Transactions on Automatic Control, vol. 55, no. 9, pp. 2030-2042, Sept 2010.

[5] K.-E. Årzén, "A simple event-based pid controller," in Proceedings of the 14th World Congress of IFAC, Beijing, March 1999, pp. 423-428.

[6] S. Durand and N. Marchand, "Further results on event-based pid controller," in Proceedings of the European Control Conference (ECC), 2009.

[7] S. Durand, J. Minet, J. Castellanos, and N. Marchand, "Asyncar, a radio-controlled vehicle for asynchronous experiments implementation of an event-based cruise control," in Proceedings of the 8th International Conference on Electrical Engineering Computing Science and Automatic Control (CCE), Oct 2011, pp. 1-6.

[8] W. P. M. H. Heemels, J. H. Sandee, and P. P. J. Van Den Bosch, "Analysis of event-driven controllers for linear systems," International Journal of Control, vol. 81, no. 4, pp. 571-590, 2008.

[9] F. L. Roubieu, F. Expert, G. Sabiron, and F. Ruffier, "Two-directional 1 -g visual motion sensor inspired by the fly's eye," Sensors Journal, IEEE, vol. 13, no. 3, pp. 1025 -1035, march 2013.

[10] S. Viollet, L. Kerhuel, and N. Franceschini, "A 1-gram dual sensorless speed governor for micro-air vehicles," in Proceedings of the 16th Mediterranean Conference on Control and Automation, 25-27 June 2008, pp. 1270-1275. 